\title{
Adrenocorticotropic Hormone-producing Neuroendocrine Tumors of Thymus: Case Series and Review of Literature
}

\author{
${ }^{1}$ Roma Pradhan, ${ }^{2}$ Sushil Gupta, ${ }^{3}$ Niraj Kumari, ${ }^{4}$ Amit Agarwal
}

\section{ABSTRACT}

Since the first description of thymic carcinoid as a specific entity in 1972 by Rosai and Higa, approximately 92 cases of adrenocorticotropic hormone (ACTH)-producing thymic neuroendocrine associated with Cushing's syndrome (CS) have been described between 1980 and 2011. We report here three new cases of neuroendocrine tumor (NET) of thymus associated with ectopic production of ACTH along with review of recent literature.

All our three cases were middle age ( 2 male and 1 female) with clinical features of CS. Two of the three patients had hyperpigmentation. All the three underwent transsternal excision of thymic tumor.

The NET of the thymus associated with CS can occur at any age from 4 to 64 years; however, meta-analysis revealed that majority of cases occur between 18 and 40 years. There is no sex predilection for these tumors except for the NETs which are associated with MEN-1, which occurred predominantly in males.

Majority of these patients would present with clinical features of CS, although the severity and rapidity of onset would vary.

The ACTH-producing NET is a rare cause of CS and requires high suspicion to make an early diagnosis and is a locally aggressive disease that requires aggressive surgical resection. Adjuvant radiotherapy may be beneficial.

Keywords: Adrenocorticotropic hormone, Cushing's syndrome, Neuroendocrine tumors.

How to cite this article: Pradhan R, Gupta S, Kumari N, Agarwal A. Adrenocorticotropic Hormone-producing Neuroendocrine Tumors of Thymus: Case Series and Review of Literature. World J Endoc Surg 2018;10(1):1-4.

Source of support: Nil

Conflict of interest: None

${ }^{1}$ Assistant Professor, ${ }^{2,4}$ Professor, ${ }^{3}$ Additional Professor

${ }^{1}$ Department of Endocrine Surgery, Dr Ram Manohar Lohia Institute of Medical Sciences, Lucknow, Uttar Pradesh, India

${ }^{2}$ Department of Endocrinology, Sanjay Gandhi Postgraduate Institute of Medical Sciences, Lucknow, Uttar Pradesh, India

${ }^{3}$ Department of Pathology, Sanjay Gandhi Postgraduate Institute of Medical Sciences, Lucknow, Uttar Pradesh, India

${ }^{4}$ Department of Endocrine Surgery, Sanjay Gandhi Postgraduate Institute of Medical Sciences, Lucknow, Uttar Pradesh, India

Corresponding Author: Roma Pradhan, Assistant Professor Department of Endocrine Surgery, Dr Ram Manohar Lohia Institute of Medical Sciences, Lucknow, Uttar Pradesh, India Phone: +919732221166, e-mail: drromapradhan@yahoo.com

\section{INTRODUCTION}

Since the first description of thymic carcinoid as a specific entity by Rosai and Higa, ${ }^{1}$ approximately 92 cases of ACTH-producing thymic neuroendocrine associated with CS have been described between 1980 and 2011. As thymic carcinoids have a significant degree of cellular atypical and a relatively poor prognosis, they have been reclassified as thymic neuroendocrine carcinomas ${ }^{2}$ to distinguish them from pulmonary, pancreatic, and stomach carcinoids, which tend to be less aggressive. We report here three new cases of NET of thymus associated with ectopic production of ACTH along with the review of recent literature.

\section{CASE REPORTS}

\section{Case 1}

A 40-year-old lady presented with generalized body swelling along with weight gain of $16 \mathrm{~kg}$ in last 1 year. She had amenorrhea and darkening of skin for past 1 year. She also complained of fatigability, difficulty in climbing stairs, and getting up from squatting position for the past 6 months. She was also detected to be having diabetes mellitus and hypertension for the past 4 months. There was no h/o headache or visual disturbances. Family history was not contributory.

On examination, the patient was obese, had rounded face with dark pigmentation. Skin was hyperemic and petechiae were present over the legs. Her blood pressure was 146/94 $\mathrm{mm} \mathrm{Hg}$ and rest of the general examination was normal. Striae were present over the lower part of the abdomen. She had grade IV muscle power in lower limbs.

Investigations revealed anemia, but all other hematological and biochemical parameters were normal. Basal serum cortisol was $916 \mathrm{nmol} / \mathrm{L}$ (n: 110-520). The ACTH level was $143 \mathrm{pg} / \mathrm{mL}$. Overnight dexamethasone suppression test (ONDST) revealed unsuppressible cortisol level of $793 \mathrm{nmol} / \mathrm{L}$. High dose dexamethasone suppression test was nonsuppressible as well. Ultrasonography abdomen did not reveal any adrenal tumor; however, computed tomography (CT) thorax revealed an anterior mediastinal mass (Fig. 1). Magnetic resonance imaging (MRI) pituitary was normal. Inferior petrosal sinus sampling (IPSS) done was not suggestive of a pituitary 


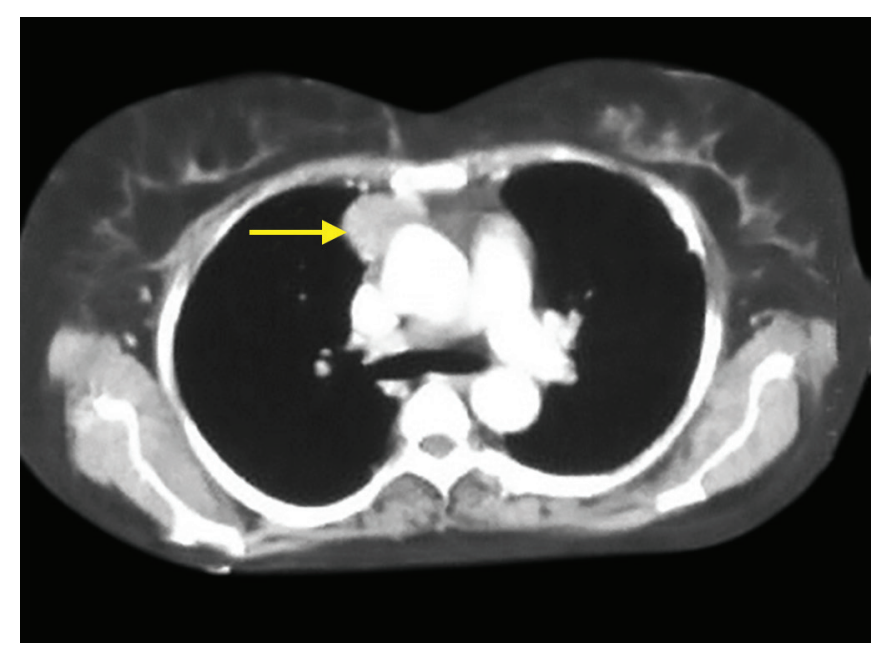

Fig. 1: Anterior mediastinal mass (arrow)

source. Hence, a diagnosis of thymic carcinoid as an ectopic source of ACTH secretion was made.

Thymic mass was removed trans-sternally. The tumor was present in the right limb and body of thymus with brachiocephalic, pleural, and pericardial infiltration (Fig. 2). Tumor was excised along with portion of brachiocephalic vein with primary repair. Histopathology report showed NET (intermediate grade). The patient is doing well in follow-up without recurrence of symptoms.

\section{Case 2}

A 36-year-old gentleman was referred to us with anterior mediastinal mass on CT thorax and with a fine-needle aspiration cytology, suggesting an NET. The patient gave history of generalized weakness, weight gain with episodic history of facial flushing. He was also detected to be having hypertension and diabetes of recent onset. He also had multiple episodes of breathlessness which required

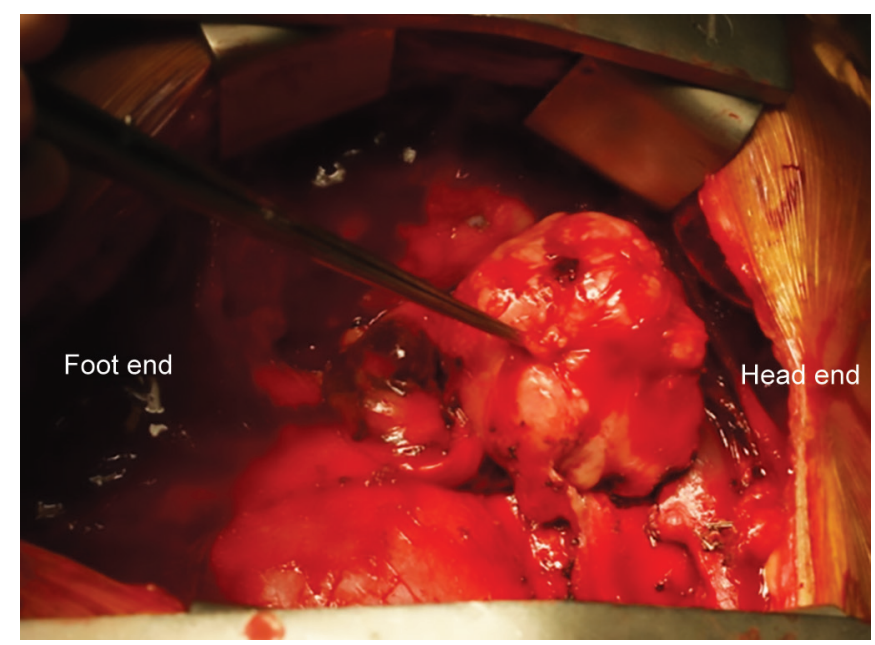

Fig. 3: Tumor adherent to brachiocephalic vein, reaching up to aortopulmonary window

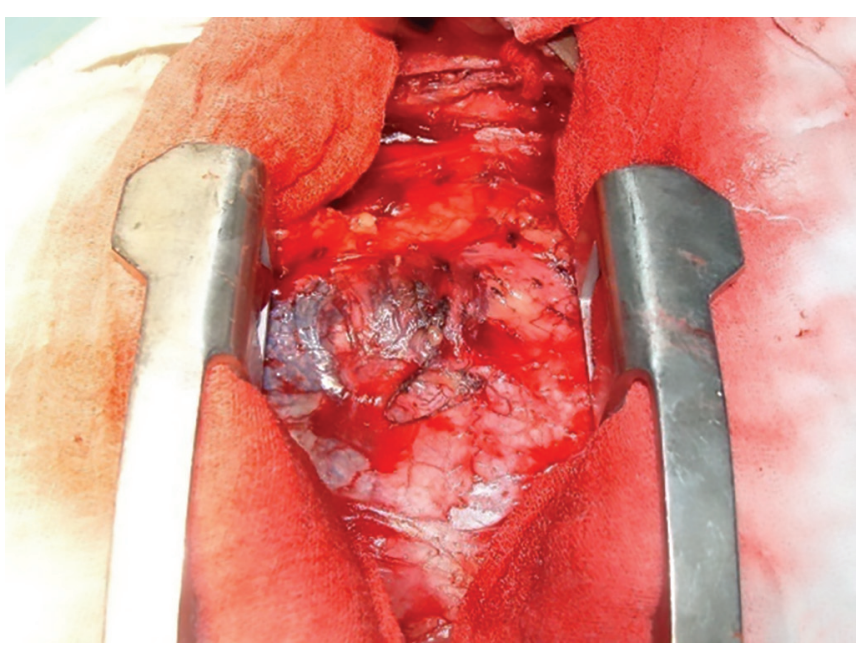

Fig. 2: Tumor arising from the body and right limb of the thymus with pleural and pericardial infiltration

admission. On examination, a clinical diagnosis of CS was made. Biochemically, ONDST was found to be nonsuppressible, and ACTH level was $108.1 \mathrm{pg} / \mathrm{mL}$. Hence, a diagnosis of ACTH (ectopic)-dependent CS was made.

Patient underwent trans-sternal excision of mass along with thymectomy. The tumor was present in the body of thymus with infiltration of brachiocephalic vein from which the mass could be dissected off (Fig. 3). Postoperative recovery was smooth. Histopathology showed intermediate-grade NET (Fig. 4) with immunohistochemistry positive for synaptophysin, chromogranin, neuronspecific enolase, CD-56, and cytokeratin with Ki-67 index being $12 \%$. The patient is recurrence free for last 3 years.

\section{Case 3}

A 40-year-old gentleman presented to our outpatient department with chest pain and exertional dyspnea of 2 months duration. The patient was evaluated at local

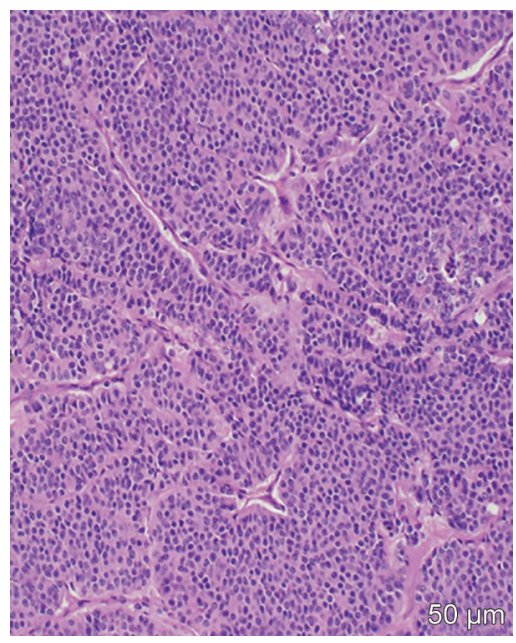

Fig. 4: Well-encapsulated tumor with nests and cords separated by thin fibrovascular septa with cells round to oval with fine stippled chromatin, inconspicuous nucleoli, and moderate cytoplasm 


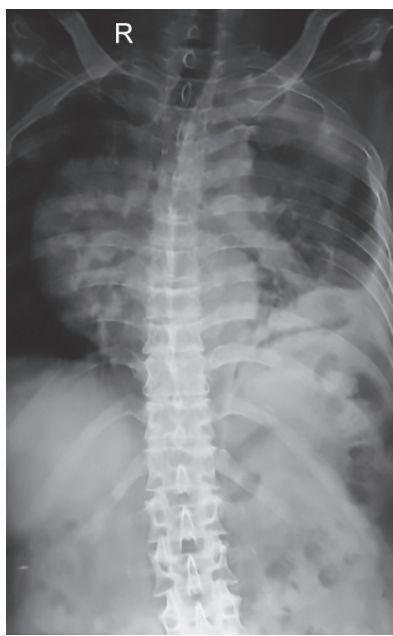

Fig. 5: X-ray showing mediastinal widening

hospital where chest X-ray showed mediastinal widening (Fig. 5), and CT chest showed a large anterior mediastinal mass (Fig. 6) and with that the patient was referred to our department. On detailed history taking, there was h/o weight gain, facial puffiness, edema for 4 months. On examination, there was moon facies, dilated veins over neck, striae over thigh, hyperpigmentation confined to hands, feet, and face, with severe proximal muscle weakness. Hence, a diagnosis of hypercortisolism with anterior mediastinal mass was made. Biochemical investigation showed unsuppressed ONDST and low dose dexamethasone suppression test and raised ACTH. In the pituitary, MRI brain showed a suspicious hypodense lesion; therefore, ultrasound-guided fine needle aspiration was planned for thymic mass, which was suggestive of neuroendocrine neoplasm. Final diagnosis of ectopic ACTH syndrome due to a thymic carcinoid was made. After adequate preoperative preparation and under steroid cover, the patient was taken up for surgery. A median sternotomy was performed and the tumor which was in the region of body of thymus was removed. The weight of specimen was 320 gm. Postoperatively, patient required ventilatory support for 3 days. Though the patient was shifted out of intensive care unit, he expired after 3 days due to cardiorespiratory failure.

\section{DISCUSSION}

Ectopic ACTH-secreting tumors occur primarily in the lungs, pancreas, esophagus, and stomach. In the study of 80 cases of NETs of the thymus by Moran and Suster, ${ }^{2}$ 18 patients $(22 \%)$ had endocrine manifestations, while 20 patients (25\%) were completely asymptomatic. A metaanalysis in 2012 described 92 cases of ACTH-producing thymic NET associated with $\mathrm{CS}^{3}$ followed by 12 cases of National Institutes of Health series ${ }^{3}$ and 7 cases from India. ${ }^{4}$ Moreover, NET of the thymus associated with CS

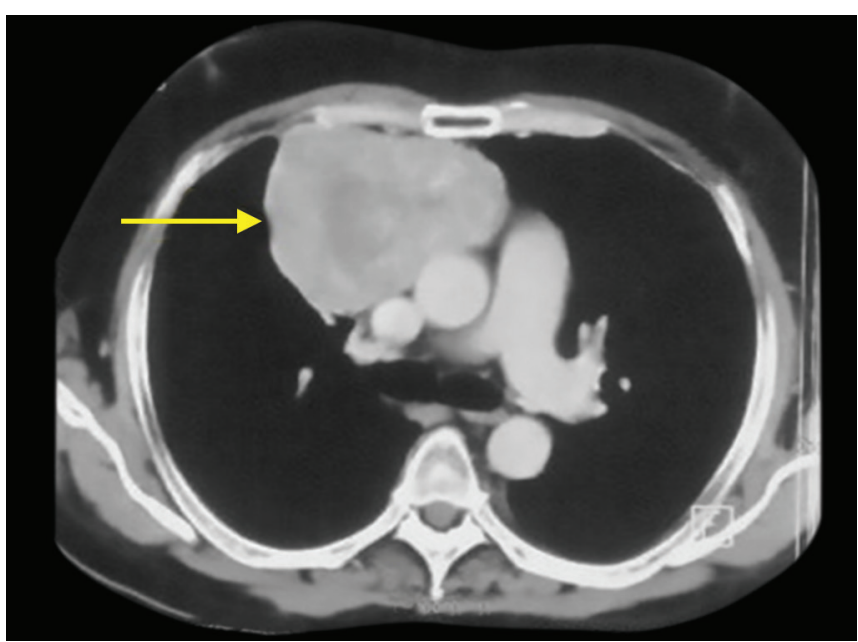

Fig. 6: Anterior mediastinal mass

can occur at any age from 4 to 64 years; however, metaanalysis $^{3}$ revealed that majority of cases occur between 18 and 40 years. There is no sex predilection for these tumors except for the NETs which are associated with MEN-1, which occurred predominantly in males.

Majority of these patients would present with clinical features of CS, although the severity and rapidity of onset would vary. Hyperpigmentation can be a striking feature. Neary et $\mathrm{al}^{3}$ reported hyperpigmentation in $50 \%$ of their 12 cases. In our series, two to three cases presented with hyperpigmentation.

It has been observed by de Perrot et $a 1^{5}$ that the time interval between the first clinical complaint and diagnosis of thymic NEC varied between 6 months and 8 years with a mean of 3 years. ${ }^{6,7}$ This variation could be because of intermittent secretion of ACTH. The diagnosis of CS is made by unsuppressible levels of serum cortisol. Elevated levels of ACTH exclude the possibility of an autonomous adrenal tumor. The next challenge is to differentiate between a pituitary adenoma and an ectopic source of ACTH. The most efficient way to differentiate between them is to perform IPSS.

Once an ectopic ACTH source is confirmed, localization is done by contrast-enhanced CT (CECT) chest with $5 \mathrm{~mm}$ slices. Functional imaging in the form of octreotide radiolabeled scan can be done, since these tumors are known to express somatostatin receptors in $80 \%$ of cases. In contrast with bronchial carcinoids which usually remain occult, thymic ACTH-producing NET can be easily identified on chest imaging. In the series of Neary et $\mathrm{al}^{3}$ a thymic tumor was identified in 9 of 11 cases on initial chest imaging (chest X-ray or CECT chest). Although thymic NETs are rarely part of MEN-1, the possibility of MEN-1 should be considered in all patients with thymic NET, especially in male smokers. ${ }^{8}$ Preoperatively, patient may be put on short-term medical therapy 
like ketoconazole, octreotide, aminoglutethimide, mifepristone. Surgery in the form of total thymectomy is the therapy of choice for these tumors. Since aggressive local resection is required, limited approach should be avoided. Median sternotomy is thus indicated in almost all cases. These tumors can be locally aggressive and aggressive resection may include pericardium and pleura. Pass et $\mathrm{al}^{9}$ have suggested that surgery should include aggressive mediastinal lymph node dissection. In the series by Neary et $\mathrm{al}^{3}{ }^{3} 10$ patients underwent thymectomy via sternotomy; 2 patients underwent thymectomy via thoracotomy. En bloc resection of pericardium was required in 2 patients and excision of pleura in 1 patient. In our series, all the 3 patients underwent thymectomy via median sternotomy.

Carcinoid tumors have been reclassified (World Health Organization) as:

Grade I (well differentiated)

Grade II (well differentiated)

Grade III (poorly differentiated).

Majority of thymic carcinoids are grade II. ${ }^{10,11}$

Immunohistochemistry analysis usually shows positive staining with chromogranin A and synaptophysin. Presence of ACTH staining does not necessarily correlate with presence or absence of CS.

Need for adjuvant radiotherapy or chemotherapy is still not clear. Ruffini et $\mathrm{al}^{12}$ noted that postoperatively radiation therapy may reduce the risk of relapse but has no effect on long-term survival. These tumors are resistant to standard chemotherapy regimes. In the series by Neary et $\mathrm{al}^{3}{ }^{3} 3$ patients received fractionated mediastinal radiation therapy, 4 also received adjuvant chemotherapy. The behavior of these tumors appears to correlate with histologic grade, which seems directly proportional to degree of differentiation. Despite total thymectomy or aggressive local resection, these tumors carry a poor prognosis and most patients present with local recurrence or metastasis within 5 years after surgery with overall survival of 10 years. In the meta-analysis done by Neary et $\mathrm{al}^{3}{ }^{3}$ median time to recurrence of CS in 53 patients was 18 months, with median time to death being 35 months. Thymic NET associated with CS has a poor outcome as compared with those without CS. ${ }^{13}$ Control of recurrent CS can be done using ketoconazole, metyrapone, or bilateral adrenalectomy, the latter is preferred to highly toxic mitotane. In the meta-analysis the longest disease-free interval was 8 years. ${ }^{14}$ Therefore, all patients of NET associated with CS should have extended clinical follow-up with intensive biochemical testing and imaging.

\section{CONCLUSION}

In summary, ACTH-producing NET is a rare cause of CS and requires high suspicion to make an early diagnosis and is a locally aggressive disease which requires aggressive surgical resection. Adjuvant radiotherapy may be beneficial.

\section{REFERENCES}

1. Rosai J, Higa E. Mediastinal endocrine neoplasm of probable thymic origin, related to carcinoid tumor. Clinicopathologic study of 8 cases. Cancer 1972 Apr;29(4):1061-1074.

2. Moran CA, Suster S. Neuroendocrine carcinomas (carcinoid tumor) of the thymus. A clinicopathologic analysis of 80 cases. Am J Clin Pathol 2000 Jul;114(1):100-110.

3. Neary NM, Lopez-Chavez A, Abel BS, Boyce AM, Schaub N, Kwong K, Stratakis CA, Moran CA, Giaccone G, Nieman LK. Neuroendocrine ACTH-producing tumor of the thymus experience with 12 patients over 25 years. J Clin Endocrinol Metab 2012 Jul;97(7):2223-2230.

4. Sathyakumar S, Paul TV, Asha HS, Gnanamuthu BR, Paul MJ, Abraham DT, Rajaratnam S, Thomas N. Ectopic Cushing syndrome: a 10-year experience from a tertiary care center in southern India. Endocr Pract 2017 Aug;23(8):907-914.

5. de Perrot M, Spiliopoulos A, Fischer S, Totsch M, KeshavjeeS. Neuroendocrine carcinoma (carcinoid) of the thymus associated with Cushing's syndrome. Ann Thorac Surg 2002 Feb;73(2):675-681.

6. McCaughey ES, Walker V, Rolles CJ, Scheurmier NI, Hale AC, Rees LH. Ectopic ACTH production by a thymic carcinoid tumour. Eur J Pediatr 1987 Nov;146(6):590-591.

7. Lin KL, Chen CY, Hsu HH, Kao PF, Huang MJ, Wang HS. Ectopic ACTH syndrome due to thymic carcinoid tumor in a girl. J Pediatr Endocrinol Metab 1999 Jul-Aug;12(4):573-578.

8. Ghazi AA, Dezfooli AA, Mohamadi F, Yousefi SV, Amirbaigloo A, Ghazi S, Pourafkari M, Berney D, Ellard S, Grossman AB. Cushing syndrome secondary to a thymic carcinoid tumor due to multiple endocrine neoplasia type 1. Endocr Pract 2011 Jul-Aug;17(4):e92-e96.

9. Pass HI, Doppman JL, Nieman L, Stovroff M, Vetto J, Norton JA, Travis W, Chrousos GP, Oldfield EH, Cutler GB Jr. Management of the ectopic ACTH syndrome due to thoracic carcinoids. Ann Thorac Surg 1990 Jul;50(1):52-57.

10. Klemm KM, Moran CA. Primary neuroendocrine carcinomas of the thymus. Semin Diagn Pathol 1999 Feb;16(1):32-41.

11. Wick MR. Neuroendocrine neoplasia. Current concepts. Am J Clin Pathol 2000 Mar;113(3):331-335.

12. Ruffini E, Oliaro A, Novero D, Campisi P, Filosso PL. Neuroendocrine tumors of the thymus. Thorac Surg Clin 2011 Feb;21(1):13-23.

13. Wick MR, Scott RE, Li CY, Carney JA. Carcinoid tumor of the thymus: a clinicopathologic report of seven cases with a review of the literature. Mayo Clin Proc 1980 Apr;55(4):246-254.

14. Takagi J, Otake K, Morishita M, Kato H, Nakao N, Yoshikawa K, Ikeda H, Hirooka Y, Hattori Y, Larsson C, et al. Multiple endocrine neoplasia type I and Cushing's syndrome due to an aggressive ACTH producing thymic carcinoid. Intern Med 2006 Feb;45(2):81-86. 